\title{
EATERDAD Intervención familia-aula para el desarrollo de habilidades grafomotrices
}

\author{
Family-classroom intervention for the development of \\ graphomotor skills
}

Dra. Yanet Amanda Maquera-Maquera es docente e investigadora de la Universidad Nacional del Altiplano (Perú) (ymaquera@unap.edu.pe) (https://orcid.org/0000-0002-2492-6428).

(D) Dr. Saúl Bermejo-Paredes es docente e investigador de la Universidad Nacional del Altiplano (Perú) (sbermejo@unap.edu.pe) (https://orcid.org/0000-0001-9885-7974).

(D) Erika Olivera-Condori es docente del Instituto Superior Pedagógico Público de Puno (Perú) (erika_olco@hotmail.com) (https://orcid.org/0000-0001-9315-6853).

Recibido: 2020-06-09 / Revisado: 2020-12-02 / Aceptado: 2020-12-08 / Publicado: 2021-01-01

\section{Resumen}

La complejidad de las acciones pedagógicas para el profesorado de educación inicial, no se reduce a dirigir y dar pautas desde un pedestal; es parte de la clase, requiere de mucho tacto y preparación. Cada vez, el esfuerzo es mayor para innovar y generar procesos de aprendizaje estimulantes y productivos; sin embargo, paralelamente las expectativas de los padres y la sociedad son muy altas. Situación que convoca a transformar las prácticas pedagógicas y responder a las necesidades sociales y de aprendizaje de los niños. Los objetivos del estudio son: a) Describir las creencias de los educadores y padres de familia respecto a la iniciación de la alfabetización temprana en niños de cinco años de edad; y b) Establecer en qué medida el modelo de involucramiento familiar a través de la realización de actividades de motricidad fina desde los hogares, favorece el aprendizaje de las habilidades grafomotrices. La metodología empleada corresponde al enfoque mixto, de alcance descriptivo-explicativo. Los resultados evidencian no solo el impacto del modelo de involucramiento familiar, sustentado en la incorporación e intercambio de experiencias y conocimientos entre docentes de aula y padres de familia, en el aprendizaje de la alfabetización temprana; más bien resaltan que tomar en cuenta las creencias de los docentes y padres de familia son decisivas para transformar y cambiar la trayectoria de lo cotidiano y usual en la cultura educativa.

Descriptores: Aprendizaje-servicio, aprendizaje cooperativo, creencias pedagógicas, enseñanza de la escritura, influencia familiar, psicomotricidad.

\section{Abstract}

The complexity of pedagogical actions for initial education teachers is not reduced to directing and giving guidelines from a pedestal; it is part of the class, it requires a lot of tact and preparation. Every time, the effort is greater to innovate and generate stimulating and productive learning processes, however; at the same time, the expectations of parents and society are very high. Situation that calls for transforming pedagogical practices and responding to the social and learning needs of children. The objectives of the study are: a) to describe the beliefs of educators and parents regarding the initiation of early literacy in children of 05 years of age; and b) establish to what extent the family involvement model through the performance of fine motor activities from home, favors the learning of graphomotor skills. The methodology used corresponds to the mixed approach, descriptive-explanatory in scope. The results show not only the impact of the family involvement model, based on the incorporation and exchange of experiences and knowledge between classroom teachers and parents, in the learning of early literacy; Rather, they highlight that taking into account the beliefs of teachers and parents are decisive to transform and change the trajectory of the everyday and usual in the educational culture.

Keywords: Service-learning, cooperative learning, pedagogical beliefs, teaching of writing, family influence, psychomotor skills.

Forma sugerida de citar: Maquera-Maquera, Y.A., Bermejo-Paredes, S., \& Olivera-Condori, E., (2021). Intervención familia-aula para el desarrollo de habilidades grafomotrices. Alteridad, 16(1), 92-104. https://doi.org/10.17163/alt.v16n1.2021.07 


\section{Introducción}

La grafomotricidad es un proceso comunicativoevolutivo que se encarga de los pre-aprendizajes de la comunicación de signos gráficos (Suárez, 2004); aplica una serie de habilidades psicomotoras en los niños para prepararlos en el aprendizaje de la escritura, y constituye, un buen elemento para la interiorización de imágenes parciales de las letras (Alfonso et al., 2012; Lurçat, 1988; Segura et al., 2017); es la psicomotricidad aplicada como paso previo al acto de escribir, consistente en actividades como el garabato, producción de trazos, dibujo libre, sobre cualquier superficie (Ramón, 2016; Reynoso, 2019; Sugrañes et al., 2007), que se emplean en la educación preescolar, con el propósito de iniciar fructíferamente la alfabetización temprana. La adquisición y el dominio de la lengua escrita es una tarea trascendental en el desarrollo madurativo de todo niño y el reto más significativo para todo sistema escolar (Gómez-Díaz et al., 2015), es uno de los aprendizajes más difíciles y complejos, vinculado al manejo y aprendizaje de otras habilidades — por lo mismo- exige cierta madurez o condiciones necesarias para enfrentarlo y evitar el miedo, fracaso y frustración (Fornaris, 2011).

La educación infantil es el período más crítico y de mayor relevancia capital para el bienestar futuro en el desarrollo de la persona; las experiencias y procesos de socialización vividos serán las bases de las habilidades para aprendizajes ulteriores, configurar su identidad, autoestima y conocer el mundo que lo rodea (Balongo \& Mérida, 2017; Pastor, 2018; UNESCO, 2014). Esto implica tener conocimientos profundos sobre la atención temprana de la infancia y sus propósitos; conocer cada individualidad; develar el talento de cada niño para actuar y crear alternativas de respuesta a sus necesidades y problemas complejos. La educación inicial en el Perú se concibe como el nivel que sienta las bases para la formación integral de los niños menores de seis años, articulada de manera pedagógica y curricular con la educación primaria (MINEDU,
2017); dedica buena parte del tiempo lectivo a dar sentido y significado al desarrollo de las competencias comunicativas, en todas sus manifestaciones. Uno de los objetivos principales de la educación inicial constituye, la alfabetización (Lara \& Pulido, 2020), la adquisición y dominio de la lengua escrita (Gutiérrez \& Díez, 2015), que se ha convertido en prerrequisito para la promoción escolar.

La adquisición preescolar de la representación escrita del lenguaje es una valiosa habilidad que mejora los resultados de la alfabetización temprana de los niños (Gerde et al., 2019; Hall et al., 2015); no obstante, requiere previamente del desarrollo de las habilidades grafomotoras globales (Cisternas et al., 2014), que asociado a esto están los "primeros garabatos, en los que el niño no está dibujando el objeto en sí, sino que está fijando en el papel los gestos con los que él mismo representa a dicho objeto" (Montealegre, 2006, p. 26). Estas representaciones gráficas como manifestación del desarrollo motor fino están vinculadas a la "capacidad de usar la mano y los dedos de manera precisa, de acuerdo con la exigencia de la actividad y se refiere a las destrezas necesarias para manipular un objeto" (Serrano \& Luque, 2019, p. 16). De este modo, la escritura a mano sirve para vincular el procesamiento visual con la experiencia motora, facilitando las habilidades de reconocimiento de letras posteriores (James, 2017). Por consiguiente, la práctica de la grafomotricidad a mano es más fructífera respecto a otras alternativas (Grabowski, 2010); en este sentido Benítez y Sánchez (2018), concluyen en efecto que "educar el gesto gráfico desde el movimiento motriz es una alternativa potente en Educación Infantil porque facilita la resolución del problema de aprendizaje del trazado y la escritura" (p. 195).

La educación inicial a diferencia de otras modalidades y niveles de educación básica, no enseña contenidos temáticos, ni trata a los niños como estudiantes en situación escolar. No obstante, las posibilidades de un aprendizaje eficiente de la escritura frecuentemente, son limitadas 
en los entornos preescolares (Gerde et al., 2019); persisten las prácticas pedagógicas rutinarias que siguen un itinerario mecánico, preestablecido, poco propicio para enriquecer la actuación y configuración del andamiaje de la expresión y comunicación lingüística, que debe fundamentarse en el conocimiento metalingüístico (Arnaíz \& Bolarín, 2016), como capacidad para reconocer la naturaleza, formas y funciones del lenguaje escrito. Crear alternativas de respuesta a necesidades y problemas complejos de los niños preescolares, exigen a los docentes cada vez, mayor adaptabilidad e innovación en el empleo de diversas estrategias comunicativas en cada episodio interactivo, variando de forma cualitativa y cuantitativa (Gonzáles, 2015). Por lo contrario, el fracaso en la alfabetización temprana de los niños preescolares, se mantiene invariablemente, y cuando esto ocurre, se pierde el objetivo del aprendizaje de la escritura como una "herramienta de vida de cada ser humano y pasa a convertirse en un problema de aprendizaje" (Suárez, 2004, p. 6), que obstruye y retarda el proceso de aprendizaje del lenguaje escrito y la comunicación, en general.

La adquisición de la representación escrita del lenguaje por los niños ha sido vista como un aprendizaje que sólo corresponde al sistema escolar (Ferreiro, 2006); sin embargo, en la etapa preescolar los niños, reciben una mayor injerencia de sus entornos y contextos inmediatos, compuesta de diversos factores y en coparticipación de diversos agentes educativos, quienes deben crear estímulos adecuados en las condiciones favorables para potenciar el desarrollo (Gutiérrez et al., 2018). El aprendizaje temprano de la alfabetización traspasa los muros escolares, se da en diversos contextos, tanto formales como informales de aprendizaje (Parodi, 2010). Ante esta circunstancia, se plantea concebir escuelas infantiles no solo pensado para los niños, exclusivamente, sino centradas en familias, construyendo relaciones complementarias a partir del respeto, confianza y encuentro mutuos entre la familia y escuela (Ferrer \& Riera, 2015; Keyser,
2006). Bajo este modelo de involucramiento, se reconoce que los docentes establecen relaciones más fluidas y dinámicas con los padres de los niños, para que cooperen con la concreción de actividades específicas asignadas en la escuela, por cuanto son quienes conocen mejor las particularidades de cada niño y pueden influir enormemente en el desarrollo y bienestar de sus hijos y poder convertirse en un eje para asegurar la calidad de la educación preescolar (Mir et al., 2009; Pastor, 2018).

Así, la decadencia de las prácticas pedagógicas tradicionales de alfabetización temprana, abre paso hacia formas más democráticas y respetuosas de enseñanza, con intervención conjunta de educadores, comunidad educativa y la familia de los niños para promover capacidades comunicativas funcionales en todas sus manifestaciones, como base de un desarrollo integral (Díaz, 2019; Palos et al., 2017; Ramos, 2011). En esta perspectiva, el estudio tiene por objetivos: a) Describir las creencias de los educadores y padres de familia respecto a la iniciación de la alfabetización temprana en niños de cinco años de edad; y b) Establecer en qué medida el modelo de involucramiento familiar a través de la realización de actividades de motricidad fina favorece el aprendizaje de habilidades en niños preescolares de cinco años de edad.

Se trata de construir propuestas de aprendizaje sustentadas en acciones auténticas-participativas y experiencias del ámbito escolar y familiar, para atender articuladamente, las necesidades de aprendizaje de los niños y satisfacer las expectativas de la comunidad, y juntos contribuir a la transformación de nuestros contextos escolares, culturales y sociales. El estudio se inscribe en la perspectiva de la pedagogía participativa, de aprendizaje en servicio, considerado como propuesta pedagógica, programa, estrategia (Deeley, 2016; Mendia, 2012; Puig et al., 2007), que combina de manera activa y participativa procesos de aprendizaje y servicio a la comunidad, aportando un escenario formativo que potencia las habilidades de comunicación, 
actitudes sociales y la motivación requerida para la concreción de las tareas y competencias previstas (Chiva-Bartoll et al., 2018; Gil et al., 2016; Huda et al., 2018).

\section{Metodología}

\subsection{Ubicación temporal-espacial}

Estudio realizado durante el segundo semestre del año escolar 2019, en la Institución Educativa Inicial (IEI) San Martín de Porres, ciudad de Puno. Corresponde al distrito, provincia y departamento de Puno-Perú.

\subsection{Muestra}

Constituida por el total de niños y padres de familia, sección única de cinco años, IEI San Martín: a) 18 niños (ocho varones y diez mujeres); b) 18 padres de familia (15 mujeres y tres varones); y c) tres docentes, todas mujeres (las docentes de las secciones de tres y cuatro años, sólo participaron durante las entrevistas en profundidad). Para la selección de la muestra primaron dos criterios: la predisposición y cooperación de la maestra de aula, permitiendo que uno del equipo investigador asuma en aula el desarrollo de las sesiones experimentales en su lugar; $y$, el consentimiento e involucramiento real de los padres de familia en la propuesta pedagógica. Además, es necesario señalar que los padres de familia de esta sección de niños, constituían el grupo mejor organizado y destacado al interior de la institución educativa.

\subsection{Enfoque y alcance}

Por la naturaleza y propósitos explicitados es un estudio de caso, con enfoque mixto: cualitativocuantitativo y de alcance descriptivo-explicativo, ejecutado a través de 12 sesiones de aprendizaje tanto en aula como en los hogares de los niños, con una periodicidad de dos horas semanales ininterrumpidamente, durante el segundo semestre del año escolar 2019.

\begin{tabular}{|c|c|c|c|}
\hline $\begin{array}{l}\text { GRAFOMO- } \\
\text { TRICIDAD } \\
\text { (dimensiones) }\end{array}$ & $\begin{array}{l}\text { EJECUCIÓN DE } \\
\text { HABILIDADES }\end{array}$ & CRITERIOS DE EVALUACIÓN & $\begin{array}{l}\text { ESCALA DE } \\
\text { VALORACIÓN }\end{array}$ \\
\hline $\begin{array}{l}\text { Manipulativo- } \\
\text { vivencial }\end{array}$ & $\begin{array}{l}\text { Trazos sincré- } \\
\text { tico tensos y } \\
\text { distendidos }\end{array}$ & $\begin{array}{l}\text { Utilizando crayones de colores ejecuta garabatos, líneas } \\
\text { con angulaciones y ondulantes de manera continua. }\end{array}$ & \multirow{3}{*}{$\begin{array}{l}\text { Según escala de } \\
\text { evaluación de nive- } \\
\text { les de aprendizaje } \\
\text { establecida por el } \\
\text { Ministerio de Edu- } \\
\text { cación del Perú: } \\
\text { inicio C (00-10 pun- } \\
\text { tos), proceso B (11- } \\
15 \text { puntos) y logro A } \\
\text { (16-20 puntos). }\end{array}$} \\
\hline $\begin{array}{l}\text { Interiorización } \\
\text { simbólica }\end{array}$ & $\begin{array}{l}\text { Trazos linea- } \\
\text { les tensos y } \\
\text { distendidos }\end{array}$ & $\begin{array}{l}\text { El niño representa o sustituye objetos reales en objetos } \\
\text { internos al unir líneas de acuerdo con una imagen, tra- } \\
\text { za líneas del mismo tamaño y dirección, une dos pun- } \\
\text { tos a través de una línea, dibuja un objeto, otorgando } \\
\text { significados. }\end{array}$ & \\
\hline $\begin{array}{l}\text { Representación } \\
\text { perceptiva }\end{array}$ & $\begin{array}{l}\text { Trazos ico- } \\
\text { nográficos y } \\
\text { opacidades }\end{array}$ & $\begin{array}{l}\text { Representación de figuras abiertas-cerradas, formas } \\
\text { gráficas pre-esquemáticas y dibujo libre, distinguiendo } \\
\text { forma y fondo. }\end{array}$ & \\
\hline
\end{tabular}

Para describir las creencias de docentes y padres respecto a la iniciación de la alfabetización temprana en los niños preescolares, se empleó la técnica de la entrevista en profundidad previo al tratamiento experimental, considerando como categorías de análisis: a) Percepciones sobre vinculación escuela-familia (combinación de interacciones comunicativas, experiencias y conocimientos entre padres y maestros para concretar las tareas escolares y competencias de aprendizaje previstas para los niños); b) Creencias sobre alfabetización temprana y; el aprendizaje de habilidades grafomotrices durante el tratamiento cuasi-experimental en grupo único, con pre y posprueba, fueron determinados según resultados obtenidos mediante la téc- 
nica de la observación y la ficha de evaluaciónvaloración de los aprendizajes.

La ficha de evaluación-valoración, como instrumento de investigación empleada para medir el nivel de los aprendizajes en grafomotricidad, presentó básicamente la siguiente estructura:

\subsection{Procedimientos en la ejecución del modelo de intervención}

- Autorización formal y consentimiento de la dirección de institución, docente de aula y padres de familia, para ejecutar la propuesta de investigación educativa.

- Inclusión y aprobación de la propuesta bajo mecanismos de participación y consenso, durante las reuniones de trabajo compartidos entre docente de aula y padres de familia. El equipo investigador, ha delegado a un representante para proponer $y$ consensuar los propósitos, procedimientos y acciones del trabajo conjunto a implementarse, tanto al interior de aula, como en los hogares de los niños.

- La ejecución de las 12 actividades de aprendizaje en aula, bajo el modelo de intervención, estuvo a cargo de un miembro del equipo investigador. La maestra de aula y los otros investigadores asumían la observación de la clase con la finalidad de realizar un balance en el avance de la propuesta y proceder a los reajustes, si así se requerían.

- Las actividades o tareas desarrolladas por los niños en los hogares, fueron ejecutadas siguiendo un plan de procedimientos sencillos, previamente consensuados y aprobados en las reuniones de sección de cinco años, según dimensiones de la variable dependiente y los logros de aprendizaje previstos respecto a habilidades grafomotoras. Estas actividades, estaban articuladas a la secuencia metodológica desarrollada en aula, denominándose con- vencionalmente: extensión de la sesión de aprendizaje, bajo la dirección de los padres o familiares. Ante la expectativa generada y solicitud permanente de los padres, en muchas ocasiones, los miembros del equipo investigador asistieron a la ejecución de actividades en el hogar a fin de asesorar y monitorear la intervención.

- El equipo investigador sometía a evaluación semanal la marcha del experimento en todos sus componentes. Cualquier propuesta de replanteamiento, implicaba consensuar entre todos los participantes del estudio, en particular con la maestra de aula.

- La entrevista en profundidad fue realizada antes y después de las reuniones de padres y docentes, así como en los momentos de ingreso, espacios de espera y salida de los niños de la institución educativa, prioritariamente.

\subsection{Variables/categorías de análisis}

La variable independiente estuvo representada por el modelo de intervención familia-aula, tipificándose como una estrategia de aprendizajeservicio, a partir de la cual se diseñaron y ejecutaron 12 sesiones de aprendizaje, centradas en la concreción de actividades de psicomotricidad fina, considerando las dimensiones viso-motor, manual y viso-manual. La grafomotricidad como variable dependiente, tuvo por dimensiones de estudio a los niveles: manipulativo-vivencial (trazos sincréticos tensos y distendidos); interiorización simbólica (trazos lineales tensos y distendidos); y representación perceptiva (trazos iconográficos y realización de opacidades), que fueron medidos por la escala de aprendizaje establecida por el Ministerio de Educación del Perú: inicio C (00-10), proceso B (11-15) y logro A (16-20).

Por otra parte, las creencias descritas por los padres de familia y docentes, respecto a la alfabetización temprana en niños de cinco años, constituyen la categoría de análisis abordada 
desde la perspectiva cualitativa. Para este efecto, los datos recogidos fueron codificados del siguiente modo: $\mathrm{D}=$ docente y $\mathrm{F}=$ padre de familia, agregándosele un número entre paréntesis para diferenciar cada miembro de la población. Ejemplo: $\mathrm{D}(1)$ identifica a la primera docente. En el caso de los padres de familia la numeración llega hasta 18.

\subsection{Análisis de datos}

Los datos cuantitativos se analizaron mediante el software SPSS, determinándose la t calculada y el p-valor específico, en relación con el nivel de significación correspondiente, conforme se ofrece en la sección de resultados. Para los datos cualitativos se recurrió al análisis de contenido y el procedimiento de interpretación consistió básicamente en considerar la frase como unidad de análisis, para asegurar la fiabilidad de la comprensión e interpretación de los conceptos o significados. La hipótesis estadística planteada fue:

$$
\begin{aligned}
& \mathrm{Ho}: \mu_{2}=\mu_{1} \\
& \mathrm{H} a: \mu_{2}>\mu_{1}
\end{aligned}
$$

\section{Resultados}

El estudio ofrece resultados de valor pedagógico y social a través de datos y reflexiones cualitativos (creencias sobre iniciación en alfabetización temprana) y cuantitativos (aprendizaje de habilidades grafomotrices a través del modelo de intervención pedagógica familia-escuela), que guardan una estrecha relación entre sí: coincidiendo con los resultados de otros estudios, las creencias o subjetividades constituyen un espacio de construcción de significados y sentidos que dirigen las acciones y modos de ser de los individuos (Izaguirre \& Alba, 2016); las creencias o subjetividades que elaboran los docentes orientan su trabajo, influyen y modifican sus prácticas pedagógicas, son fundamentales en la toma de decisiones curriculares y concreción de los objetivos de aprendizaje, integran nuevos aprendizajes en situaciones prácticas y finalmen- te, las subjetividades guían las acciones y toma de decisiones en los docentes (Cuadra et al., 2015; Gómez et al., 2014; Hernández-Álvarez, 2010).

\subsection{Creencias de padres de fami- lia y docentes sobre iniciación en alfabetización temprana de niños de cinco años de edad}

Las docentes de educación inicial, consideran que la articulación familia-escuela, es un asunto de corresponsabilidad, para garantizar y contribuir a una adecuada formación integral y educación de calidad de los niños preescolares; sin embargo — - según sus creencias pedagógicasiniciar a los niños de cinco años en habilidades para la escritura no es lo usual; sin embargo, se muestran con apertura hacia nuevas situaciones y propuestas de enseñanza-aprendizaje.

Tanto los padres y nosotros como docentes tenemos la obligación de integrarnos, estar más cerca de nuestros niños, ambos somos los responsables de su educación. (D-1)

La educación de los niños no es responsabilidad exclusiva de la maestra, sino sobre todo también de los padres. (D-3)

Definitivamente, en esta institución y en toda la educación inicial producto de nuestras reuniones pedagógicas, hemos ya aclarado que educación inicial no se dedica a la enseñanza de la lectoescritura, vayamos a ver qué sucede con la propuesta, estoy inquietada, parece distinta. (D-2)

Por su parte, los padres de familia admiten que es necesario mantener vínculos de comunicación permanente con la maestra de aula y la institución educativa, para conocer de cerca los progresos y dificultades de aprendizaje en los niños; y tienen expectativas de ver a sus niños que hayan adquirido las competencias básicas en lectoescritura, al culminar la educación inicial a los cinco años de edad. 
Tenemos que estar siempre comunicados. Nos interesa que nuestros niños sepan más, mejor si la maestra se esfuerza por enseñarles a leer y escribir. (F-8)

Muy de acuerdo la comunicación, sino cómo nos enteraríamos de lo que aprenden los niños, ahora sería una gran cosa que nuestros niños empiecen y terminen la promoción sabiendo escribir algo. (F-13)

Los padres estamos por nuestros niños, siempre estaremos identificados con la institución y dispuestos a participar en todo. (F-1)

Es más juego y poco aprendizaje de conocimientos, yo aplaudiría si los niños terminaran escribiendo. (F-18)

Sí, claro sin comunicarnos con la maestra, no estaríamos enterados con lo que pasa en la institución, es muy importante. (F-5)

Algunas percepciones de los padres de familia, señalan a la institución educativa como una organización independiente y arbitraria a sus intereses, además, atribuyen a los educadores el papel de la enseñanza y trabajo con los niños; no obstante, se muestran colaborativos con la organización de padres y la maestra de aula.

La institución nunca consulta con los padres, sólo nosotros nos dedicamos a cumplir lo que ellos mandan, pero ahí estamos, cualquier asunto lo asumimos, no nos negamos. (F-4)

Si de enseñar se trata, eso corresponde a las docentes, nosotras podíamos tal vez apoyar con algo. (F-6)
Las creencias pedagógicas de las educadoras respecto al vínculo docente-padre de familia, privilegian el sentido de mayor apertura y permeabilidad entre ambas partes, sustentada en relaciones respetuosas recíprocas, fundamentalmente. Además, enfatizan que los padres deben ser parte de los procesos de retroalimentación en casa, situación que implica un trabajo cooperativo entre padres y docentes, a favor de los aprendizajes de los niños.

Deben entender que nosotras y ellos los padres, modelamos a los niños, tenemos que ser flexibles y guardar respeto entre nosotros. (D-1)

Ellos, los padres deben reforzar aquello que hacemos con los niños en el aula, porque a nosotras los niños, no nos ven exactamente como docentes. (D-2)

\subsection{Modelo de involucramien- to para el desarrollo de la grafomotricidad}

El modelo de intervención pedagógica interactivo familia-aula, diseñado en la perspectiva metodológica de la estrategia aprendizaje-servicio, centrado en la ejecución de actividades psicomotoras finas - a través de un tratamiento cuasi-experimental - ha permitido incrementar los niveles de aprendizaje en las habilidades grafomotoras manipulativo-vivencial, interiorización simbólica y representación perceptiva de los niños de cinco años de edad. Y como tal, según estos resultados se acepta la hipótesis alterna rechazándose la nula, conforme es evidenciable en la siguiente tabla acompañado por el p-valor y la regla de decisión correspondiente. 
Tabla 1. Resultados prueba de muestras independientes niveles de grafomotricidad en niños de cinco años

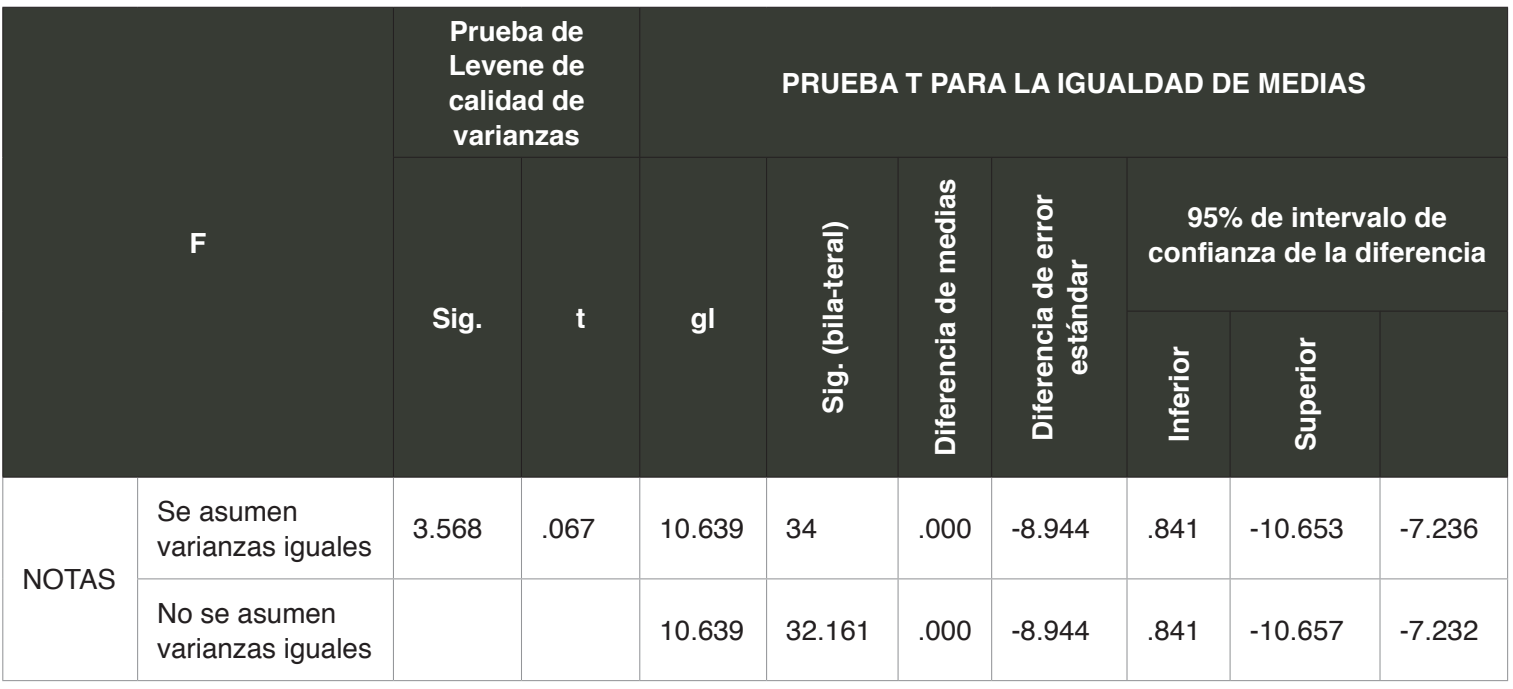

Fuente: Elaboración por autores, según datos primitivos analizados en SPSS

\section{Determinación de p-Valor}

$P$ Valor $=\frac{\text { Sig }}{2}$

\section{Regla de decisión}

Como $P$ Valor $<\alpha$ entonces se rechaza $H o$ y se acepta $\mathrm{Ha}$

Como $0<0.05$ entonces se rechaza $H o$ y se acepta $\mathrm{Ha}$

Los resultados del estudio son concordantes con otros trabajos similares de investigación que, confirmaron la eficacia de programas de intervención psicomotriz, visuomotor en el desarrollo de la grafomotricidad (Gonzáles, 2015; Layes et al., 2019); sin embargo, atribuir una certeza total a los mismos es discutible, requieren intervenciones de mayor rigurosidad científica. El diseño de investigación cuasi-experimental sin grupo control, es una limitante de validez interna que impide atribuir el grado en que ciertamente la manipulación de la variable independiente, es responsable de los cambios registrados en los aprendizajes de habilidades grafomotoras.

Lo que sí queda claro después del trabajo aplicativo, aun cuando no formaron parte de los propósitos del estudio, son las bondades del modelo de intervención pedagógica familiaaula como estrategia de aprendizaje-servicio (Mendía, 2016; Tapia, 2010): los niños trabajan colaborando interactivamente con sus padres y profesores para maximizar sus propios aprendizajes; se generan situaciones en las cuales se esperan que ocurran formas particulares de interacción para generar el logro de un aprendizaje (Collazos \& Mendoza, 2009); implica para los docentes renovar los procesos pedagógicos habituales, establecer relaciones más cercanas, dialógicas y afectivas con los niños, padres de familia y la comunidad en general, para concretar las aspiraciones relacionadas con la calidad de los aprendizajes y la educación; se renuevan los roles convencionales de docentes y estudiantes mediante transacciones colaborativas-interactivas y consensuadas, orientadas al logro de metas establecidas (Revelo-Sánchez et al., 2018).

\section{Discusión y conclusiones}

La familia y la escuela constituyen los espacios más privilegiados para construir y fructificar el desarrollo integral de los niños, ninguna de ellas 
por sí sola, de manera aislada y diferenciada puede acompañar, satisfacer y potenciar las capacidades de aprendizaje y los talentos de los niños, con calidad y pertinencia; se requiere, de la cooperación permanente y oportuna entre ambas partes, definitivamente. No obstante, en nuestro contexto las estrategias para fomentar los vínculos entre familia-escuela y comunidad son aún frágiles, limitadas y tradicionales que conllevan a plantear y redefinir el rol de la educación en general (Bermejo et al., 2020; Razeto, 2018); de tal modo, que la escuela no sea una esperanza de bienestar, sino el lugar de disfrute permanente, desde la cual se vive la vida (Bermejo \& Maquera, 2019); persiste la tendencia a valorar los procesos de alfabetización temprana, el aprendizaje de la lectoescritura en general, como prácticas relacionadas solo con la escuela (Vance et al., 2007), regularmente.

De acuerdo con los resultados obtenidos, el modelo de involucramiento familiar, escuelahogar, es una estrategia de aprendizaje-enseñanza factible y aceptable (Goldman et al., 2019), para acompañar y seguir los procesos de aprendizaje de las habilidades grafomotrices en niños de cinco años de edad, reconoce la colaboración activa de los padres en el apoyo a los niños, con la ejecución de tareas específicas asignadas por los docentes (Pastor, 2018); se constituye en un espacio privilegiado de participación y aprendizaje, metodología indispensable en los procesos formativos familiares (Mendoza \& Zúñiga, 2017); por tanto, ostenta un valor pedagógico y social.

Su implementación y ejecución depende previamente, de las actitudes y creencias positivas tanto de los padres de familia como de los docentes. Comprender las creencias de los maestros y padres respecto a cómo contribuir en la construcción de los aprendizajes y conocimientos en los niños, es crucial; por cuanto informan a sus prácticas de valiosas habilidades para apoyar los aprendizajes reales (Gerde et al., 2019); actúan como filtros del conocimiento adquirido que permite a las personas entender el mundo y desenvolverse en él de una determinada manera (Díaz, 2013); de lo contrario -como refieren Patiño y Rojas (2009) — una pedagogía que niegue la subjetividad no es pedagogía, porque negaría al sujeto como constructor de representaciones significativas culturales, sociales e individuales.

Las estrategias de vinculación familiaaula, sustentadas en la ejecución de actividades de psicomotricidad fina, según los resultados de aprendizaje obtenidos por la mayoría de niños comprendidos en el tratamiento experimental, permiten el incremento o desarrollo de las habilidades de grafomotricidad. Los niños pueden maximizar su propio aprendizaje, interactuando a través de actividades colaborativas junto a sus padres y docentes; los padres de familia participan activamente y asumen co-responsabilidad en los procesos formativos de los niños en la escuela; y los docentes adoptan nuevos roles, establecen relaciones más dialógicas y afectivas con los niños y sus padres, implicándolos en las aspiraciones de concretar las metas educativas. En efecto, diferentes estudios realizados, confirman que el desarrollo de las habilidades grafomotoras en los niños, están basados en la adquisición de la motricidad fina (Gonzáles, 2015), que existe una estrecha relación entre motricidad fina y escritura (Lica et al., 2010); proponiéndose por consiguiente, la ejercitación fina y gruesa para el aprendizaje del grafismo (Segura et al., 2017), que incluso paulatinamente, será útil para aumentar la identificación y el reconocimiento de las letras (Gil et al., 2012; Zemloc et al., 2018). No obstante, se debe tener en cuenta las limitaciones de rigor científico-metodológico del estudio, explicitadas en la sección de los resultados.

El modelo experimentado resultó estimulante y desafiante para el aprendizaje de las habilidades grafomotrices, parte por establecer conexiones de confianza y comunicación más fluidas entre la realidad escolar y la cotidianidad de la vida de los niños. Logra que el niño realice por cuenta propia, acciones válidas y correctas de ejecución gráfica en el espacio que debe representar, manteniendo control postural adecuado, dominio del brazo y la manera cómo debe sostener el lápiz. 
Es más, el procedimiento implicado contribuye a materializar ideas que sugieren actividad y responsabilidad en los niños para configurar su estilo de vida (Ortiz, 2012). El niño al desarrollar el sentido del movimiento y la representación de manera autónoma y reflexiva, no se expone a la clásica frase de: qué se puede y qué no se puede. Deja la imitación como procedimiento favorito en la educación preescolar, y se predispone por iniciativa propia y estímulo social desarrollar competencias propias y diversificadas que lo identificarán como persona única y distinta.

La importancia de estos hallazgos, no solamente radica en el diseño de contextos de aprendizaje estimulantes y desafiantes para los niños y haber generado entre docentes y padres, oportunidades y espacios para participar, intercambiar y compartir experiencias y conocimientos durante el acompañamiento en los procesos formativos de los niños; sino en la comprobación de la existencia de un potencial humano (Guerrero, 2000), capaz de cambiar la trayectoria de lo cotidiano y usual. Por otra parte - se pone en evidencia que- las creencias, conocimientos, experiencias y la capacidad de adaptación hacia cambios inesperados tanto de padres y educadores respecto a la formación del niño, es la mayor fortaleza, el mejor aporte y esfuerzo en común para cambiar la cultura educativa. Es posible concretar realidades alternativas de práctica pedagógica, diseñando y participando en contextos de aprendizaje compartidos entre la escuela y la cotidianidad de la vida de los niños. Lo que los padres y la sociedad esperan de los docentes y la escuela, pueden encontrarlos en ellos mismos, al experimentar, enriquecer y producir nuevos estilos y valores de ser padres y contribuir a la formación humana, trascendiendo espacios y límites.

\section{Referencias bibliográficas}

Alfonso, S., Deaño, M., Almeida, L., Conde, Á., \& García-Señorán, M. (2012). Facilitación del conocimiento alfabético en preescolar a través del entrenamiento en codificación, gra- fomotricidad y lectura. Psicothema, 24(4), 573-580. https://bit.ly/31Rjhth

Arnaíz, P., \& Bolarín, M.J. (2016). Introducción a la psicomotricidad. Editorial Síntesis S. A.

Balongo, E., \& Mérida, R. (2017). Proyectos de trabajo: una metodología inclusiva en Educación Infantil. Revista Electrónica de Investigación Educativa, 19(2), 125-142.https://bit.ly/36LJs8L

Benítez, M., \& Sánchez, C. (2018). Procesos educativos en educación infantil mediante el grafismo y la escritura. Enseñanza \& Teaching. Revista Interuniversitaria de Didáctica, 36(2), 195-214. https://bit.ly/3qyK8pO

Bermejo, S., Maquera, Y.A., \& Bermejo., L.Y. (2020). Procesos de educación intercultural y autosegregación indígena en los aimaras de Puno-Perú. Revista Historia de la Educación Latinoamericana, 23(34). https://bit.ly/39OVFLA

Bermejo-Paredes, S., \& Maquera-Maquera, Y. (2019). Interpretación de la escuela rural andina en comunidades aimaras de Puno-Perú. Revista Electrónica Educare, 23(2), 1-15. https://bit.ly/37HBtsv

Cisternas, Y., Ceccato, R., Gil, D., \& Marí, M.I. (2014). Funciones neuropsicológicas en las habilidades de inicio a la lectoescritura. Revista INFAD de Psicología, 1 (1), 115-122. https://bit.ly/37zAfiS

Chiva-Bartoll, O., \& Gil-Gómez, J. (2018). Aprendizajeservicio universitario. Modelos de intervención e investigación en la formación inicial docente. Octaedro.

Collazos, C., \& Mendoza, J. (2009). Cómo aprovechar el "aprendizaje colaborativo" en el aula. Educación y Educadores, 9(2), 61-76. https://bit.ly/33Pxn0b

Cuadra, D., Jorquera, R., \& Pérez, M. (2015). Las teorías subjetivas del profesor acerca de su salud laboral: implicancias en la promoción de la salud preventiva en el trabajo docente. Revista Ciencia y Trabajo, 17(52), 1-6. https://bit.ly/2VGty90

Deeley, S. (2016). El aprendizaje-servicio en educación superior. Teoría práctica y perspectiva crítica. Narcea.

Díaz, C. (2013). Estudio de caso sobre las creencias de estudiantes de pedagogía en inglés respecto 
a la autoeficacia percibida y la enseñanza del idioma. Núcleo, 25(30), 41-67.

https://bit.ly/3kKoawX

Díaz, G.M. (2019). Tratamiento pedagógico de las alteraciones en la lectura y la escritura. Monografía de Licenciatura. Universidad Nacional de Educación Enrique Guzmán y Valle. Lima. https://bit.ly/3gVsK9g

Ferreiro, E. (2006). La escritura antes de la letra. CPUe, Revista de Investigación Educativa, (3), 1-52. https://bit.ly/2XZdfWC

Ferrer, M., \& Riera, M. (2015). Relaciones y encuentros con las familias en educación infantil. RELAdEI Revista Latinoamericana de Educación Infantil, 4(2), 27-42. https://bit.ly/3gTPd6V

Fornaris, M. (2011). Factores necesarios para la adquisición de la lectoescritura. Cuadernos de Educación y Desarrollo, (30), 3. https://bit.ly/3fRKCR5

Gerde, H.K., Wright, T.S., \& Bingham, G.E. (2019). Creencias de los maestros de preescolar e instrucción para la escritura. Revista Educación Docente de la Primera Infancia, 40(4), 326-351.

Gil, J., Moliner, O., Chiva, O., \& López, R. (2016). Una experiencia de aprendizaje-servicio en futuros docentes: Desarrollo de la competencia social y ciudadana. Revista Complutense de Educación, 27(1), 53-73. https://bit.ly/2VFg3GM

Gil, S.A., Deaño, M.D., Almeida, L.S., Rodríguez, A.C., \& García, M. (2012). Facilitación del conocimiento alfabético en preescolar a través del entrenamiento en codificación, grafomotricidad y lectura. Psicotema, 24(4), 573-580. https://bit.ly/31Rjhth

Goldman, S.E., Sanderson, K.A., \& Lloyd, B.P (2019). Efectos de la comunicación entre la escuela y el hogar con refuerzo implementado por los padres en el comportamiento fuera de la tarea para estudiantes con TEA. Discapacidades Intelectuales y del Desarrollo 57(2), 95-111. https://bit.ly/33Nf7EW

Gómez-Díaz, R., García-Rodríguez, A., \& CordónGarcía, J.A. (2015). APPrender a leer y escribir: aplicaciones para el aprendizaje de la lectoescritura. Education in the Knowledge Society, 16(4), 118-137.

https://doi.org/10.14201/eks2015164118137
Gómez, V., Muñoz, C., Silva, I., González, M.P., Guerra, P., \& Valenzuela, J. (2014). Creencias y oportunidades de aprendizaje en la práctica educativa en contextos de pobreza. Perfiles Educativos, 36(144), 173-188. https://bit.ly/39MFMFm

Gonzáles, J. (2015). Las competencias de comunicación en el inicio de la lectoescritura. Revista Brasileira de Estudios Pedagógicos, 96(243), 416-438. https://bit.ly/3mLZgxP

González, M.E. (2015). La escritura en primer año de la escuela primaria y la psicomotricidad. Revista Psicomotricidad, Movimiento y Emoción, (1), 1-14. https://bit.ly/2PMxZwj

Grabowski, J. (2010). Speaking, writing and memory span in children: Ouput modality affects cognitive performance. International Journal of Psychology, 45(1), 28-39. https://doi.org/10.1080/00207590902914051

Guerrero, L. (2000). Educación inicial: a la búsqueda del tesoro escondido. ¿Cómo enfocar la misión de la educación inicial hacia la emergencia y el florecimiento del inmenso potencial humano? Revista Iberoamericana de Educación, 22, 75-92. https://bit.ly/31OCwnl

Gutiérrez, D., Socorro, A., \& Ruiz, M. (2018). Impacto de la educación inicial y preescolar en el neurodesarrollo infantil. IE Revista de investigación educativa de la REDIECH, 9(17), 33-51. https://bit.ly/2Yf3AeP

Gutiérrez, R., \& Díez, A. (2015). Aprendizaje de la escritura y habilidades de conciencia fonológica en las primeras edades. Bordón. Revista de Pedagogía, 67(4), 43-59.

https://bit.ly/3gcTQJI

Hall, A.H., Simpson, A., Guo, Y., \& Wang, S. (2015). Examinando los efectos de la instrucción de escritura preescolar en las habilidades emergentes de alfabetización: una revisión sistemática de la literatura. Alfabetización, Investigación e Instrucción, 54(2), 115-134. https://bit.ly/3kEUUaV

Hernández-Álvarez, J.L., Velázquez-Buendía, R., Martínez, M. E., \& Díaz del Cueto, M. (2010). Creencias y perspectivas docentes sobre objetivos curriculares y factores determinantes de actividad física. Revista Internacional de Medicina y Ciencias de la Actividad Física y el Deporte, 10(38), 336-355. 
https://bit.ly/2VI08Yj

Huda, M., Shukri, K., Hisyam, N., \& Mohd, B. (2018). Transmitir la responsabilidad cívica basada en el liderazgo: conocimientos del aprendizaje mediante servicio. Revista Internacional de Ética y Sistemas, 34(1), 20-31. https://bit.ly/3qsEnKl

Izaguirre, R., \& Alba, D. (2016). Reflexiones sobre el papel de la subjetividad en el proceso docente-educativo. MULTIMED, 20(2), 437448. https://bit.ly/3osXUrY

James, Kh. (2017). La importancia de la experiencia de escritura a mano en el desarrollo del cerebro alfabetizado. Direcciones Actuales en Ciencias Psicológicas, 26(6), 502-508.

Keyser, J. (2006). De padres a socios: Construyendo un programa de primera infancia centrado en la familia. NAEYC y Redleaf Press.

Lara, P.A., \& Pulido, O. (2020). Escritura como práctica de sí y escuela rural. Praxis \& Saber, 11(25), 21-45. https://bit.ly/33NqRat

Layes, S., Chouchani, M.S., Mecheri, S., Lalonde, R., \& Rebaï, M. (2019). Eficacia de una intervención basada en visuomotor para niños con discapacidades de lectura y ortografía: un estudio piloto. Revista Británica de Educación Especial, 46(3), 317-339.

Lica, M.M., Ruiz, D.L., \& González, A.P. (2010). Relación entre ejecuciones deficientes de motricidad fina con dificultades de escritura: Análisis de un caso. Revista de Educación y Desarrollo, (13), 17-23. https://bit.ly/2PQ01qG

Lurçat, L. (1988). Pintar, dibujar, escribir, pensar. El grafismo en el preescolar. Cincel-Kapelusz.

Mendía, R. (2016). El aprendizaje-servicio una metodología para la innovación educativa. Convives, 16, 20-26. https://bit.ly/2VKNrMe

Mendoza, E., \& Zúñiga, M. (2017). Factores intra y extra escolares asociados al rezago educativo en comunidades vulnerables. Alteridad. Revista de Educación, 12(1), 79-91. https://bit.ly/3qqIt5s

MINEDU (2017). Programa Curricular de Educación Inicial. https://bit.ly/33VCNaX

Mir, M., Batle, M., \& Hernández M. (2009). Contextos de colaboración familia-escuela durante la primera infancia. Revista Electrónica de Investigación e Innovación Educativa y

Socioeducativa, 1(1), 45-58.

https://goo.gl/dGD1Kb

Montealegre, R. (2006). Desarrollo de la lectoescritura: adquisición y dominio. Acta Colombiana de Psicología, 9(1), 25-40. https://bit.ly/30TvCy5

Ortiz, M.E. (2012). Currículo y competencia. ¿Qué sujeto infantil están produciendo? Alteridad. Revista de Educación, 7(1), 50-57. https://doi.org/10.17163/alt.v7n1.2012.04

Palos, M.Ú., Avalos, M.L., Flores, F., \& Montes, R. (2017). Creencias de madres y docentes sobre el aprendizaje de la lectoescritura en Educación Preescolar. Revista Actualidades Investigativas en Educación, 17(3), 168-189. https://bit.ly/3lLBIrv

Parodi, G. (2010). Saber leer. Instituto Cervantes Aguilar.

Pastor, R. (2018). Construyendo escuela juntos. Estrategias de vinculación entre la familia y la escuela. Educación y Futuro. Revista de investigación aplicada y experiencias educativas, (39), 89-116. https://bit.ly/3anBYc1

Patiño, L., \& Rojas, H.M. (2009). Subjetividad y subjetivación de las prácticas pedagógicas en la universidad. Educación y Educadores, 12(1), 93-105. https://bit.ly/2IgDx1O

Puig, J.M., Batlle, R., Bosch, C., \& Palos, J. (2007). Aprendizaje servicio. Educar para la ciudadanía. Editorial Octaedro.

Ramón, M. (2016). El papel de la grafomotricidad como paso previo a la escritura. (Tesis maestría). Departamento de Educación. Universidad Jaume I. Castellón-España. https://bit. ly/33WTcMn

Ramos, M. (2011). El problema de comprensión y producción de textos en el Perú. Revista Digital de Investigación en Docencia Universitaria, 5(1), 30-53. https://doi.org/10.19083/ridu.5.5

Razeto, A. (2018). Estrategias para promover la participación de familias en la educación de niños en escuelas chilenas. Educação e Pesquisa, 44, 1-20. https://bit.ly/2LfNEoZ

Revelo-Sánchez, O., Collazos-Ordoñez, C.A., \& Jiménez-Toledo, J.A. (2018). El trabajo colaborativo como estrategia didáctica para la enseñanza/aprendizaje de la programación: Una revisión sistemática de literatu- 
ra. Tecnológicas, 21(41), 115-134. https://bit. ly/3lKUrTZ

Reynoso, M. (2019). XI Congreso Internacional de Investigación y Práctica Profesional en Psicología. XXVI Jornadas de Investigación. $\mathrm{XV}$ Encuentro de Investigadores en Psicología del MERCOSUR. I Encuentro de Investigación de Terapia Ocupacional. I Encuentro de Musicoterapia. Facultad de Psicología-Universidad de Buenos Aires, Buenos Aires. https://bit.ly/33T7uNX

Segura, M., Sabaté, M., \& Caballé, C. (2017). La psicomotricidad, un recurso para la mejora del grafismo en educación infantil. Aportaciones de la psicomotricidad al grafismo. Revista Iberoamericana de Psicomotricidad y Técnicas Corporales, (42), 5-19. https://bit.ly/30S07En

Serrano, P., \& Luque, C. (2019). Motricidad fina en niños y niñas. Desarrollo, problemas, estrategias de mejora y evaluación. Narcea, S.A.

Suárez, B. (2004). El desafío de la escritura: en busca de la grafomotricidad. Revista Iberoamericana de Psicomotricidad y Técnicas Corporales, (16), 5-16.
Sugrañes, E., Àngel, À.M., Neus, M. Antón, M., Colomé, J., Martí, M.T., Martín, R.M., Pinell, M., Rodríguez, N., Yuste, M., \& Yuste, R. (2007). La educación psicomotriz (3-8 años). Cuerpo, movimiento, percepción, afectividad: $U$ propuesta teórico práctica. Graó.

Tapia, M.N. (2010). La propuesta pedagógica del 'Aprendizaje-Servicio': Una perspectiva latinoamericana. Tzhoecoen, Revista Científica, $5,23-43$.

UNESCO (2014). El Informe de seguimiento de la EPT en el mundo. Enseñanza y aprendizaje: lograr la calidad para todos. UNESCO. https://bit.ly/31QbxrK

Vance, C., Smith, P.H., \& Murillo, L.A. (2007). Prácticas de lectoescritura en padres de familia. Influencias en el desarrollo de la lectoescritura de sus hijos. Revista Latinoamericana de Lectura, 28(3), 6-15. https://bit.ly/3kH7zKq

Zemloc, D., Vinci-Booher, S., \& James, Kh. (2018). La producción de símbolos visuales-motores facilita el reconocimiento de letras en niños pequeños. Leyendo y Escribiendo, 31(6), 1255-1271. 